\title{
Vector Games with Potential Function
}

\author{
Lucia Pusillo id \\ DIMA-Department of Mathematics, University of Genova, via Dodecaneso 35, 16146 Genova, Italy; \\ lucia.pusillo@unige.it
}

Received: 1 August 2017; Accepted: 15 September 2017; Published: 21 September 2017

\begin{abstract}
The theory of non cooperative games with potential function was introduced by Monderer and Shapley in 1996. Such games have interesting properties, among which is the existence of equilibria in pure strategies. The paper by Monderer and Shapley has inspired many game theory researchers. In the present paper, many classes of multiobjective games with potential functions are studied. The notions of generalized, best-reply and Pareto potential games are introduced in a multicriteria setting. Some properties and Pareto equilibria are investigated.
\end{abstract}

Keywords: multiobjective games; Pareto equilibria; generalized potential; best reply potential; Pareto potential

\section{Introduction}

In general, potential scalar games have an attractive feature in common: every maximizer of the potential function, a real valued function on strategy profile, is an equilibrium ( $N E$ for short) for the game. It is natural to ask if the same is valid for multiobjective games, also called vector games, with the suitable changes, considering Pareto equilibria instead of Nash equilibria and defining suitable best-reply correspondences.

This problem was partially investigated in [1,2]. In the present paper, we consider some classes of games with potential function.

The potential function is similar to a payoff function of one agent who chooses the strategies for all players.

In 1973, Rosenthal ([3]) introduced the class of congestion games which have one equilibrium in pure strategies, if they are finite. Some years later, in 1996, Monderer and Shapley ([4]) introduced potential games (exact, ordinal and generalized). They proved that the exact potential games have interesting relations with the games introduced by Rosenthal, and all potential games have at least an equilibrium in pure strategies: the maximum of a potential function corresponds to an equilibrium of the potential game.

In previous papers $([1,2])$, exact and ordinal potential games in a multicriteria setting were studied. The goal of studying vector case is to have more applications in real life. In fact, the decision makers have not one but several objectives "to maximize", which are often not comparable.

Taking into account the properties studied in Vector Optimization (see [5]), we can study which properties from the optimization problems can be generalized to mathematical vector games.

From the pioneering paper of Monderer and Shapley, potential games, due to their desirable properties, were adopted for many mathematical models, such as radio resource management for wireless communications systems and networking (see [6] and references therein). In [7,8] potential games were applied to allocation in wireless data network. In [9] the author considers generalized potential games which is an important class of generalized Nash equilibrium problems. Some methods of finding solutions for these games and Pareto equilibria of some multiobjective problems are proposed. Generalized potential games and algorithms have been studied in [10]. 
In [11], potential games were applied to environmental problems where the cooperation among players is partial. To reduce pollution, not all the countries agree and therefore a partial cooperative game arises.

The problem of approximate equilibria for infinite potential games was studied in [1]. An innovative concept which captures simultaneously the idea of exact and approximate equilibria was introduced in [12].

In [13], the authors investigated if the well-posedness property of a potential game is equivalent to the well-posedness property as maximum problem of the corresponding potential function. Many other classes of potential games were considered in the literature as generalized, best-reply potential, Nash potential (see $[14,15]$ and references therein). We study these classes in a multicriteria setting, (see [16-18] for a study about multicriteria games). We investigate the finite improvement property (FIP for short), the cycle of the best reply property and the relations between the equilibria of a potential game and those of the coordination game (where the payoff functions are equal to the potential function for each player).

The paper is organized as follows: Section 2 gives a background about results, definitions and known notations; in Sections 3-5 we study respectively generalized, best-reply, Pareto potential games in the vector case. We conclude by investigating the relations among these. In Section 6 we give some suggestions for further research. Many examples illustrate the proven properties.

\section{Background}

Given a vector $x=\left(x_{1}, \ldots, x_{n}\right) \in \prod_{i=1}^{n} X_{i}$, we write $X_{-i}=\prod_{j \neq i}^{n} X_{j}$,

$x_{-i}=\left(x_{1}, \ldots, x_{i-1}, x_{i+1}, \ldots, x_{n}\right) \in X_{-i}$ and, for all $y_{i} \in X_{i}$ and $x_{-i} \in X_{-i}$

$\left(y_{i}, x_{-i}\right)=\left(x_{1}, \ldots, x_{i-1}, y_{i}, x_{i+1}, \ldots, x_{n}\right),\left(x_{i}, x_{-i}\right)=x=\left(x_{1}, \ldots, x_{n}\right)$.

Given $x, y \in \mathbb{R}^{n}$ we consider the following inequalities on $\mathbb{R}^{n}$ :

$x \geqq y \Leftrightarrow x_{i} \geq y_{i} \quad \forall i=1, \ldots, n ;$

$x \geq y \Leftrightarrow x \geqq y$ and $x \neq y$;

$x>y \Leftrightarrow x_{i}>y_{i} \quad \forall i=1, \ldots, n$.

Analogously we define $\leqq, \leq,<$.

We write $\mathbb{R}_{++}^{m}=\left\{x \in \mathbb{R}^{m}: x_{i}>0 \forall i=1, \ldots, n\right\}$ and $\mathbb{R}_{+}^{m}=\left\{x \in \mathbb{R}^{m}: x_{i} \geq 0 \forall i=1, \ldots, n\right\}$.

We say that $U \subset \mathbb{R}^{n}$ is upper bounded (u.b. for short) if there exists $b \in \mathbb{R}^{n}$ such that $x \leq b \forall x \in U$.

For a function $F: V \subset \mathbb{R}^{n} \rightarrow \mathbb{R}^{m}$ a point $\hat{x} \in V$ is strongly Pareto optimal (sPE(F) for short) if there is no other feasible point $x$ for which $F(x)$ is larger than $F(\hat{x})$ in at least one coordinate and not smaller in all other coordinates, i.e., $\nexists x \in V$ s.t. $F(x) \geq F(\hat{x})$.

A feasible point $\hat{x} \in \mathbb{R}^{m}$ is weakly Pareto-optimal if there is no other feasible point $x$ such that $F(x)$ is larger than $F(\hat{x})$ in each coordinate, i.e., $\nexists x \in V$ s.t. $F(x)>F(\hat{x})$.

Definition 1. A strategic multiobjective game is a tuple

$$
\Gamma=\left\langle N,\left(X_{i}\right)_{i \in N},\left(u_{i}\right)_{i \in N}\right\rangle
$$

where $N$ is the set of players, $X_{i}$ is the strategy space for player $i \in N, X$ is the cartesian product $\prod_{i \in N} X_{i}$ of the strategy spaces $\left(X_{i}\right)_{i \in N}$ and each player has $m(i)$ objectives, i.e., the utility function for player $i$ is a function $u_{i}: X \rightarrow \mathbb{R}^{m(i)}$.

In general, in vector games each player $i$ may have $m(i)$ different objectives to "optimize"; the existence of a potential requires that each player has the same number of objectives: $m(i)=m$.

In previous papers, exact potential games ([1]) and ordinal potential games ([2]) in the multicriteria case were studied, so we recall them because there are some relations with other potential games which we are going to study. 
Definition 2. The strategic form of an exact potential game is a tuple $\Gamma=\left\langle N,\left(X_{i}\right)_{i \in N},\left(u_{i}\right)_{i \in N}\right\rangle$, $u_{i}: X \rightarrow \mathbb{R}^{m}$ and there exist a map $P: X \rightarrow \mathbb{R}^{m}$, such that for all $i \in N, x_{i}, y_{i} \in X_{i}, x_{-i} \in X_{-i}$, it holds

$$
u_{i}\left(x_{i}, x_{-i}\right)-u_{i}\left(y_{i}, x_{-i}\right)=P\left(x_{i}, x_{-i}\right)-P\left(y_{i}, x_{-i}\right) .
$$

Definition 3. $\Gamma=\left\langle N,\left(X_{i}\right)_{i \in N},\left(u_{i}\right)_{i \in N}\right\rangle$ with $u_{i}: X \rightarrow \mathbb{R}^{m}$ is called an ordinal potential game if there exists a map $P: X \rightarrow \mathbb{R}^{m}$ such that for all $i \in N, x_{i}, y_{i} \in X_{i}, x_{-i} \in X_{-i}$ it holds

$$
u_{i}^{j}\left(x_{i}, x_{-i}\right)>u_{i}^{j}\left(y_{i}, x_{-i}\right) \Leftrightarrow P^{j}\left(x_{i}, x_{-i}\right)>P^{j}\left(y_{i}, x_{-i}\right)
$$

for all $j=1, \ldots, m$.

We remark that when a variable is indexed by a subscript it is because we are referring to the strategy used by player $i$, while when a variable is indexed by a superscript then we refer to an objective. Shapley [19] gave a generalization of the classical definition of Nash equilibrium, the so called Pareto equilibrium (weak and strong Pareto equilibrium) for a game. This is a generalization of Nash equilibrium ( $N E$ for short) to a multicriteria setting. We will use these definitions for our multicriteria games.

Definition 4. Given a strategy profile $\hat{x}=\left(\hat{x}_{i}, \hat{x}_{-i}\right) \in X$, it is called

(a) a weak Pareto equilibrium for the multiobjective strategic game $\Gamma$ if for all $i \in N \nexists x_{i} \in$ $X_{i}$ s.t. $u_{i}\left(x_{i}, \hat{x}_{-i}\right)>u_{i}\left(\hat{x}_{i}, \hat{x}_{-i}\right)$;

(b) a strong Pareto equilibrium for the game $\Gamma$ if for all $i \in N \nexists x_{i} \in X_{i}$ s.t. $u_{i}\left(x_{i}, \hat{x}_{-i}\right) \geq u_{i}\left(\hat{x}_{i}, \hat{x}_{-i}\right)$.

The set of all strong (weak) Pareto equilibria of $\Gamma$ will be denoted by $\operatorname{PPE}(\Gamma)(w P E(\Gamma))$. We will write $P E(\Gamma)$ when we consider indifferently the strong or weak Pareto equilibria to our goals.

In other words:

Definition 5. Given a game $\Gamma=\left\langle N,\left(X_{i}\right)_{i \in N},\left(u_{i}\right)_{i \in N}\right\rangle$,

(a1) $\hat{a} \in \prod_{i \in N} X_{i}$ is called a weak Pareto equilibrium of the game if $\forall i \in N$, it turns out $\hat{a}_{i} \in w P B\left(\hat{a}_{-i}, u_{i}\right)$ which is the Pareto best reply to $\hat{a}_{-i}$ (via the function $u_{i}$ ) and it is defined in the following way:

$$
w P B\left(\hat{a}_{-i}, u_{i}\right)=\left\{a_{i} \in X_{i}: \forall b_{i} \in X_{i}, u_{i}\left(b_{i}, \hat{a}_{-i}\right) \notin u_{i}\left(\hat{a}_{i}, \hat{a}_{-i}\right)+\mathbb{R}_{++}^{m}\right\}
$$

and

(b1) $\hat{a} \in \prod_{i \in N} X_{i}$ is called a strong Pareto equilibrium of the game if $\forall i \in N$ it turns out $\hat{a}_{i} \in s P B\left(\hat{a}_{-i}, u_{i}\right)$ which is the strong Pareto best reply to $\hat{a}_{-i}$ (via the function $u_{i}$ ) and it is defined in the following way:

$$
\operatorname{sPB}\left(\hat{a}_{-i}, u_{i}\right)=\left\{a_{i} \in X_{i}: \forall b_{i} \in X_{i}, u_{i}\left(b_{i}, \hat{a}_{-i}\right) \notin u_{i}\left(\hat{a}_{i}, \hat{a}_{-i}\right)+\mathbb{R}_{+}^{m} \backslash 0\right\} .
$$

(See [1,12]).

Intuitively in finding Pareto optimal points we have to distinguish strongly and weakly Pareto optimal points.

A feasible point in $\mathbb{R}^{n}$ is strongly Pareto-optimal if there is no other feasible point which is larger in at least one coordinate and not smaller in all other coordinates.

A feasible point in $\mathbb{R}^{n}$ is weakly Pareto-optimal if there is no other feasible point which is larger in each coordinate.

\section{Generalized Potential Games}

The notion of generalized potential games in the scalar case has been given in [4] and it can be extended to a multiobjective setting. 
Definition 6. $\Gamma=\left\langle N,\left(X_{i}\right)_{i \in N},\left(u_{i}\right)_{i \in N}\right\rangle$ with $u_{i}: X \rightarrow \mathbb{R}^{m}$ is called a generalized potential game if there exists a map $P: X \rightarrow \mathbb{R}^{m}$ such that for all $i \in N, x_{i}, y_{i} \in X_{i}, x_{-i} \in X_{-i}$ it holds

$$
u_{i}^{j}\left(x_{i}, x_{-i}\right)>u_{i}^{j}\left(y_{i}, x_{-i}\right) \Rightarrow P^{j}\left(x_{i}, x_{-i}\right)>P^{j}\left(y_{i}, x_{-i}\right)
$$

for all $j=1, \ldots, m$.

In the following we will write the collection of generalized potential games with $G$.

Remark 1. In the Definition 6 of a generalized potential game we have to note that:

(a) If $u_{i}^{j}\left(y_{-i}, x\right)-u_{i}^{j}\left(y_{-i}, z\right)=0$ for some $j=1, \ldots, m$ then there is nothing we can say about the corresponding relations on $P$;

(b) If the relations about $u_{i}$ are non comparable then the corresponding relations about $P$ are not comparable (because the intuitive idea is that the strict preferences are preserved from $u_{i}$ to $P$ ).

Here is an example of a generalized potential bicriteria game:

Example 1. Let us consider the following game

$$
\begin{array}{ll|c|c|} 
& \multicolumn{1}{c}{L} & \multicolumn{1}{c}{R} \\
\cline { 3 - 4 } \Gamma_{1}: & T & (0,1)(0,0) & (1,1)(0,2) \\
\cline { 3 - 4 } & B & (0,0)(1,2) & (2,3)(2,2) \\
\cline { 3 - 4 } & & &
\end{array}
$$

where $w P E\left(\Gamma_{1}\right)=\{(T, L),(B, L),(B, R)\}$, and $\operatorname{PPE}\left(\Gamma_{1}\right)=\{(B, R)\}$. A generalized potential is equal to

$$
\begin{array}{ccc|c|} 
& \multicolumn{1}{c}{L} & \multicolumn{1}{c}{R} \\
\cline { 3 - 4 } P: & T & (0,0) & (0,2) \\
\cline { 3 - 4 } & B & (1,-1) & (2,4) \\
\cline { 3 - 4 } & &
\end{array}
$$

$w P E\left(\Gamma_{1}^{P}\right)=\{(T, L),(B, R)\}$

Another generalized potential function is the following:

$$
\begin{array}{cc|c|c|} 
& \multicolumn{1}{c}{L} & \multicolumn{1}{c}{R} \\
\cline { 3 - 4 } P_{1}: & T & (0,0) & (0,2) \\
\cline { 3 - 4 } & B & (-1,-2) & (2,3) \\
\cline { 3 - 4 } & &
\end{array}
$$

$\Gamma_{1}$ is a generalized potential game.

Note that $\operatorname{sPE}(P)=w P E(P)=\{(B, R)\}=s P E\left(P_{1}\right)=w P E\left(P_{1}\right)$.

Studying $\Gamma_{1}^{P_{1}}$ and $\Gamma_{1}^{P}$ which are the pure coordination games having the potential function as utility function, we can note that $w P E\left(\Gamma_{1}^{P}\right) \subset w P E\left(\Gamma_{1}\right)$ and $w P E\left(\Gamma_{1}^{P_{1}}\right) \subset w P E\left(\Gamma_{1}\right)$. So in general we can see that " $="$ is not valid. It is valid for other potential games.

Proposition 1. If $\Gamma$ is a generalized potential finite game then:

(1) $w P E(\Gamma) \neq \varnothing$

(2) $w P E(P) \subseteq w P E(\Gamma)$.

Proof. Let $\hat{x} \in w P E(P), w P E(P) \neq \varnothing$ being $\Gamma$ a finite game, $P$ has finite number of values. For all $i \in N$

(a) $\nexists x_{i} \in X_{i}$ s.t. $P\left(x_{i}, \hat{x}_{-i}\right)>P\left(\hat{x}_{i}, \hat{x}_{-i}\right)$ and this inequality implies that

(b) $\nexists x_{i} \in X_{i}$ s.t. $u_{i}\left(x_{i}, \hat{x}_{-i}\right)>u_{i}\left(\hat{x}_{i}, \hat{x}_{-i}\right)$

in fact if by contradiction there were $\bar{x}_{i}$ such that the inequality in (b) is valid, for the same point the inequality in (a) would be valid. So $\hat{x} \in w P E(\Gamma)$ and this proves (2) and (1). 
Proposition 2. Let $\Gamma$ be a game with $n$ players and the strategy sets be intervals in $\mathbb{R}$. Let us suppose that the utility functions are continuously differentiable. If $\Gamma$ is a generalized potential game then the following relation is valid:

$$
\frac{\partial u_{i}^{k}}{\partial x_{i}}>0 \Rightarrow \frac{\partial P^{k}}{\partial x_{i}}>0
$$

$\forall k=1, \ldots, m$ and $\forall i=1, \ldots, n$.

Proof. Starting from the definition of a generalized potential game and fixing the objective $k$, we obtain the relations between the first order derivative of $u_{i}^{k}$ and $P^{k}$ in the definition interval.

The following definition is given in [4] and adapted to a multicriteria setting

Definition 7. A finite path $\ell=\left(x_{1}, \ldots, x_{t}\right)$ in the strategy space $X$ is a finite sequence of elements $x_{r} \in X$ such that $\forall r$, the strategy combination $x_{r}$ and $x_{r+1}$ differs in the $i(r)$-th coordinates, (the player $i(r)$ is moving at step $r$ ). It is called closed or cycle if $x_{1}=x_{t}$. It is a simple cycle if it is closed and all strategy combinations are different except the initial and final point. A finite path $\left(x_{1} \ldots x_{t}\right)$ is called a weak improvement cycle if

$$
x_{1}=x_{t}
$$

$u_{i(r)}\left(x_{r}\right) \leq u_{i(r)}\left(x_{r+1}\right)$ for some $r \in 1,2, \ldots, t$.

Intuitively the path is a weak improvement cycle if it is closed and the player moving at step $r$ improves his/her payoff from the strategy $x_{r}$ to the strategy $x_{r+1}$.

A multiobjective game has the finite improvement property, (FIP for short), if every improvement path is finite.

Proposition 3. Let $\Gamma$ be a finite game. The following properties are equivalent:

(a) $\Gamma$ has the FIP

(b) $\Gamma$ has no strict improvement cycle

(c) $\Gamma$ is a generalized potential game.

Proof. The equivalence between (a) an (b) is obvious, in fact if $\Gamma$ had strict improvement cycles, then it can be run infinite times against the FIP.

For the equivalence between (a) and (c), the proof is as in [20] adapted to a multicriteria setting.

Remark 2. If a game $\Gamma$ has ordinal potential then it has generalized potential. The converse is not true as the following example proves.

Example 2. Let us consider the following example:

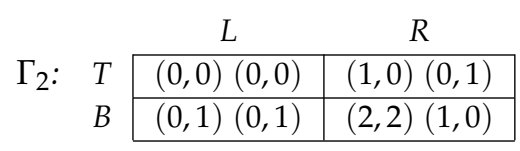

An ordinal potential is the following

$$
\begin{array}{ll|c|c|} 
& \multicolumn{1}{c}{L} & \multicolumn{1}{c}{R} \\
\cline { 3 - 4 } & T & (0,0) & (0,2) \\
\cline { 3 - 3 } & B & (0,4) & (1,3) \\
\cline { 2 - 3 } & &
\end{array}
$$

and a generalized potential (not ordinal) is for example

$$
\begin{array}{cc|c|c|} 
& \multicolumn{2}{c}{L} & \multicolumn{1}{c}{R} \\
\cline { 3 - 4 } P_{2} & T & (0,0) & (1,2) \\
\cline { 3 - 4 } & B & (0,4) & (2,3) \\
\cline { 3 - 4 } & &
\end{array}
$$


We denote the collection of ordinal potential games by $O$, it turns out $O \subset G$, and $\Gamma_{2} \in G \cap O$.

Example 3. The following game is a generalized potential but not an ordinal one:

$$
\begin{array}{cc|c|c|}
\multicolumn{1}{c}{L} & \multicolumn{1}{c}{L} \\
\cline { 3 - 4 } \Gamma_{3}: & T & (0,0)(0,0) & (0,1)(0,0) \\
\cline { 3 - 4 } & B & (0,0)(0,0) & (1,0)(0,0) \\
\cline { 3 - 4 } & &
\end{array}
$$

A generalized potential is:

$$
\begin{array}{cc|c|c|} 
& \multicolumn{2}{c}{L} & \multicolumn{1}{c}{R} \\
\cline { 3 - 4 } P_{3}: & T & (0,0) & (-1,2) \\
\cline { 3 - 4 } & B & (0,0) & (0,0) \\
\cline { 3 - 4 } & &
\end{array}
$$

$\Gamma_{3} \in G \backslash O$.

$\Gamma_{3}$ is not an ordinal potential game, in fact if $P=\left(P_{1}, P_{2}\right)$ were an ordinal potential for the game, the following relation would be valid:

$P_{1}(T, L)=P_{1}(T, R)<P_{1}(B, R)=P_{1}(B, L)=P_{1}(T, L)$ and this is impossible.

\section{Example 4. An application: a multicriteria duopoly model.}

Let us suppose that there are two companies I and II which are the producers of a certain commodity.

Both companies can decide to advertise (strategy A) their product through illustrative papers and television spots or not invest in advertisement (strategy $N A$ ).

The problem is that the printing of these panels has a bad impact in the environment of the zone because the panel-factory is highly polluting. If the factory I invests in advertisement and II does not $(A, N A)$ then I increases the value of its products by 3 and decreases the environment value by 3. At the same time the company II decreases its value products by 3 but the environment value increases by 1 . If both the companies invest in advertisement $(A, A)$, the company I will have an advantage of 1 and a loss of 2 in the environment impact and company II has an advantage of 1 for selling and a loss of 1 in the environment impact. If both companies do not invest in advertisement ( NA,NA), they obtain 3 for selling and 0 for polluting. If the company I does not invest in advertising and II does (NA, A), the company I will lose 1 for the selling but gain 1 for the environment and company II gains 3 for the products but it loses 1 for the pollution. We can make a model of this situation with the following game:

$(X, Y, f, g), f, g: X \times Y \rightarrow \mathbb{R}^{2}$ where $X=Y=\{A, N A\}$ are the strategy sets and $A$ is the strategy to invest in advertisement and $N A$ is the strategy to not invest.

$$
\begin{gathered}
f(A, N A)=(3,-3) \\
f(A, A)=(1,-2) \\
f(N A, A)=(-1,1) \\
f(N A, N A)=(3,0) \\
g(A, N A)=(-3,1) \\
g(A, A)=(1,-1) \\
g(N A, A)=(3,-1) \\
g(N A, N A)=(3,0)
\end{gathered}
$$

The strategic form of the game is:

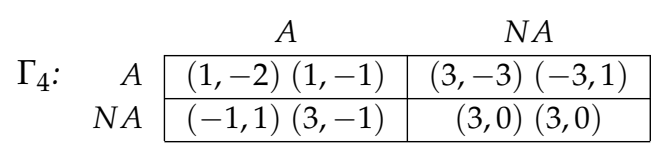

This is a generalized potential game with two criteria, a generalized potential is the following:

$$
\begin{array}{rr|c|c|} 
& \multicolumn{1}{c}{A} & \multicolumn{1}{c}{N A} \\
\cline { 3 - 3 } P_{4}: & A & (1,-1) & (0,2) \\
\cline { 3 - 3 } & N A & (0,2) & (1,3) \\
\cline { 3 - 4 } & &
\end{array}
$$

see [21] to know more about this model in the exact potential case. 


\section{Best-Reply Potential Games}

Definition 8. $\Gamma=\left\langle N,\left(X_{i}\right)_{i \in N},\left(u_{i}\right)_{i \in N}\right\rangle$ with $u_{i}: X \rightarrow \mathbb{R}^{m}$ is called a weak Best-Reply potential game (wBRP game for short) if there exists a map $P: X \rightarrow \mathbb{R}^{m}$ such that for all $i \in N, a_{-i} \in X_{-i}$ it holds

$$
w P B\left(a_{-i}, u_{i}\right)=w P B\left(a_{-i}, P\right)
$$

The function $P$ is called weak Pareto Best-Reply potential of $\Gamma$.

In a similar way we can define the strong Pareto Best-Reply potential game.

The relations have meaning in terms of components.

We will denote the collection of best reply potential games by $B R$.

Intuitively a game $\Gamma=\left\langle N,\left(X_{i}\right)_{i \in N},\left(u_{i}\right)_{i \in N}\right\rangle$ is a Pareto best reply potential game if there is a pure coordination game $\Gamma^{P}=\left\langle N,\left(X_{i}\right)_{i \in N}, P\right\rangle$, where the payoff of each player is given by function $P$, such that the best reply correspondence of each player $i$ in $\Gamma$ coincides with his (her) best response correspondence in the game $\Gamma^{P}$.

In the following we will denote the collection of Best-Reply potential games by $B R$.

Let us define a best reply cycle to illustrate some interesting properties of potential games.

Definition 9. A finite path $\ell=\left(x_{1}, \ldots, x_{t}\right)$ in the strategy space $X$ is a finite sequence of elements $x_{r} \in X$ such that $\forall r$, the strategy combination $x_{r}$ and $x_{r+1}$ differs in the $i(r)-t h$ coordinates. It is called closed or cycle if $x_{1}=x_{r}$. It is a simple cycle if it is closed and all strategy combinations are different except for the initial and final point. A path $\left(x_{1} \ldots x_{t}\right)$ is best reply compatible if the deviating player $i(r)$ moves to a best response:

$$
\forall r \quad u_{i_{(r)}}\left(x^{r+1}\right)=a \in P B\left(x_{-i(r)}^{r}, u_{i_{(r)}}\right)
$$

A finite path $\left(x_{1}, x_{2}, \ldots, x_{t}\right)$ is called a best reply cycle if it is best reply compatible and $x_{1}=x_{t}$ and for some $r \in\{1, \ldots t-1\}, u_{i(r)}\left(x_{r}\right) \leq u_{i(r)}\left(x_{r+1}\right)$

Intuitively a cycle of weak Pareto best reply (or strong Pareto best reply) is a cyclic path where in every side of the final vertex is the weak (respectively strong) Pareto best reply of the deviating player to the other's strategy.

Theorem 1. If $\Gamma$ is a finite and weak Pareto best reply potential game then $\Gamma$ has no weak Pareto best reply cycles.

Proof. Let $P$ be a $w B R$ potential for $G$ and suppose that $\left(x_{1}, \ldots, x_{m}\right)$ is a $w B R$ compatible. By the best reply compatibility $P\left(x_{k}\right)<P\left(x_{k+1}\right)$ therefore it turns out that there is $j$ such that $P^{j}\left(x_{1}\right)<\ldots .<P^{j}\left(x_{m}\right)=P^{j}\left(x_{1}\right)$ and this is a contradiction and $X$ does not contain best reply cycles.

We note that the converse is true if we define a preorder on $X$. The potential games with a preorder on the strategy space will be an argument for our next paper, for now see [15] for this topic in the scalar case.

Example 5. Let us give some examples:

$$
\begin{array}{cc|c|c|} 
& \multicolumn{1}{c}{C} & \multicolumn{1}{c}{D} \\
\cline { 3 - 4 } \Gamma_{5}: & A & (2,1)(2,0) & (0,0)(0,2) \\
\cline { 3 - 4 } & B & (0,0)(1,1) & (1,0)(0,2) \\
\cline { 3 - 4 } & &
\end{array}
$$

A Pareto Best-Reply potential is:

$$
\begin{array}{cc|c|c|} 
& \multicolumn{1}{c}{C} & \multicolumn{1}{c}{C} \\
\cline { 3 - 4 } P_{5}: & A & (3,0) & (0,2) \\
\cline { 3 - 4 } & B & (2,-1) & (1,2) \\
\cline { 3 - 4 } & &
\end{array}
$$


It turns out $w P E\left(\Gamma_{5}\right)=\{(A, C) ;(A, D) ;(B, D)\}=w P E\left(\Gamma_{5}^{P}\right) ; \operatorname{sPE}\left(\Gamma_{5}\right)=\{(A, C) ;(B, D)\}$.

This games is a $B R$ potential game and an ordinal game too, for short we will write $G_{5} \in B R \cap O$. An ordinal potential is:

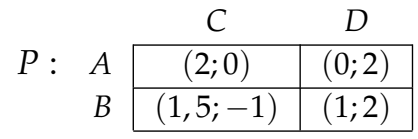

Example 6. The following is a BR-potential game but it is not an ordinal potential one:

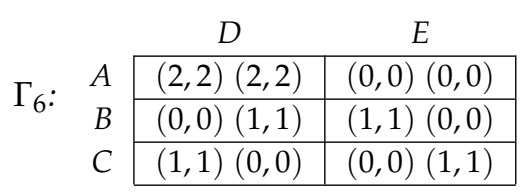

A best reply potential is:

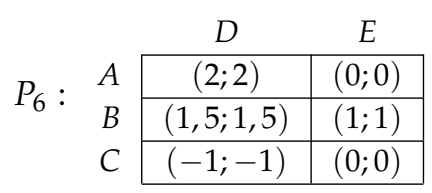

So $\Gamma_{6} \in B R \backslash O$, it is not ordinal because it has a weak improvement cycle:

$$
\gamma=\{(B, D),(B, E),(C, E),(C, D),(B, D)\} .
$$

Example 7. The following is a generalized potential game but not a best reply one.

$$
\begin{array}{cc|c|c|}
\multicolumn{1}{c}{C} & \multicolumn{1}{c}{C} \\
\cline { 3 - 4 } \Gamma_{7}: & A & (0,0)(0,0) & (0,1)(0,0) \\
\cline { 3 - 4 } & B & (0,1)(0,0) & (1,0)(0,0) \\
\cline { 3 - 4 } & & &
\end{array}
$$

$\Gamma_{7} \in G \backslash B R$, a generalized potential is:

$$
\begin{array}{lccc|} 
& \multicolumn{1}{c}{C} & \multicolumn{1}{c}{D} \\
\cline { 3 - 4 } P_{7}: & A & (0,0) & (0,0) \\
\cline { 3 - 4 } & B & (2,2) & (2,-3) \\
\cline { 3 - 4 } & &
\end{array}
$$

The game $\Gamma_{7}$ has no strict improvement cycle but this fact is not sufficient to make it a generalized potential game.

It is not a Best Reply potential game because it has a best reply cycle.

The following proposition proves some relations between the equilibria of the game and of the potential function:

Proposition 4. If $\Gamma$ is a best reply potential game and it is finite, the following relations are valid:

(1) $w P E(\Gamma) \neq \varnothing$

(2) $w P E(P) \subseteq w P E(\Gamma)$

(3) $w P E\left(\Gamma^{P}\right)=w P E(\Gamma)$

We can prove the same result for strong Pareto equilibria.

Proof. The proof is similar to that in scalar case adapting component-wise to a multicriteria setting. 


\section{Pareto Potential Games}

Definition 10. Given a game $\Gamma=\left\langle N,\left(X_{i}\right)_{i \in N},\left(u_{i}\right)_{i \in N}\right\rangle$ with $u_{i}: X \rightarrow \mathbb{R}^{m}$, it is called game with Pareto potential if there is $P: X \rightarrow \mathbb{R}^{m}$ such that

$$
w P E(\Gamma)=w P E\left(\Gamma^{P}\right)
$$

and

$$
s P E(\Gamma)=s P E\left(\Gamma^{P}\right)
$$

Note that all the potential games seen until now, except for generalized potential ones, are Pareto potential games.

We will denote the collection of Pareto potential games as $P e-P$.

\begin{tabular}{|c|c|c|c|c|}
\hline \multirow{4}{*}{$\Gamma_{8}:$} & & $D$ & $E$ & $F$ \\
\hline & $A$ & $(1,3)(1,3)$ & $(0,2)(0,2)$ & $(0,2)(0,2)$ \\
\hline & $B$ & $(0,2)(0,2)$ & $(1,3)(0,2)$ & $(0,2)(1,3)$ \\
\hline & C & $(0,2)(0,2)$ & $(0,2)(1,3)$ & $(1,3)(0,2)$ \\
\hline
\end{tabular}

Example 8. Let us consider the following bicriteria game:

$w P E\left(\Gamma_{8}\right)=\operatorname{sPE}\left(\Gamma_{8}\right)=\{(A, D)\}$.

A Pareto potential is the following:

\begin{tabular}{cc|c|c|c|}
\multicolumn{1}{c}{} & \multicolumn{1}{c}{$D$} & \multicolumn{1}{c}{$E$} & \multicolumn{1}{c}{$F$} \\
\cline { 3 - 5 }$P_{8}:$ & $A$ & $(4,4)$ & $(0,0)$ & $(0,0)$ \\
\cline { 3 - 5 } & $B$ & $(0,0)$ & $(0,0)$ & $(0,0)$ \\
\cline { 3 - 5 } & $C$ & $(3,3)$ & $(1,1)$ & $(1,1)$ \\
\cline { 3 - 4 } & & &
\end{tabular}

Note that $\Gamma_{8}$ is a Pareto potential game but not any other type of potential game. In fact, it is not a generalized potential game (and so no other types of potential) because it has a strict improvement cycle: $\gamma=\{(B, E),(B, F),(C, F),(C, E),(B, E)\}$.

The following picture shows the studied inclusions among potential multiobjective games.

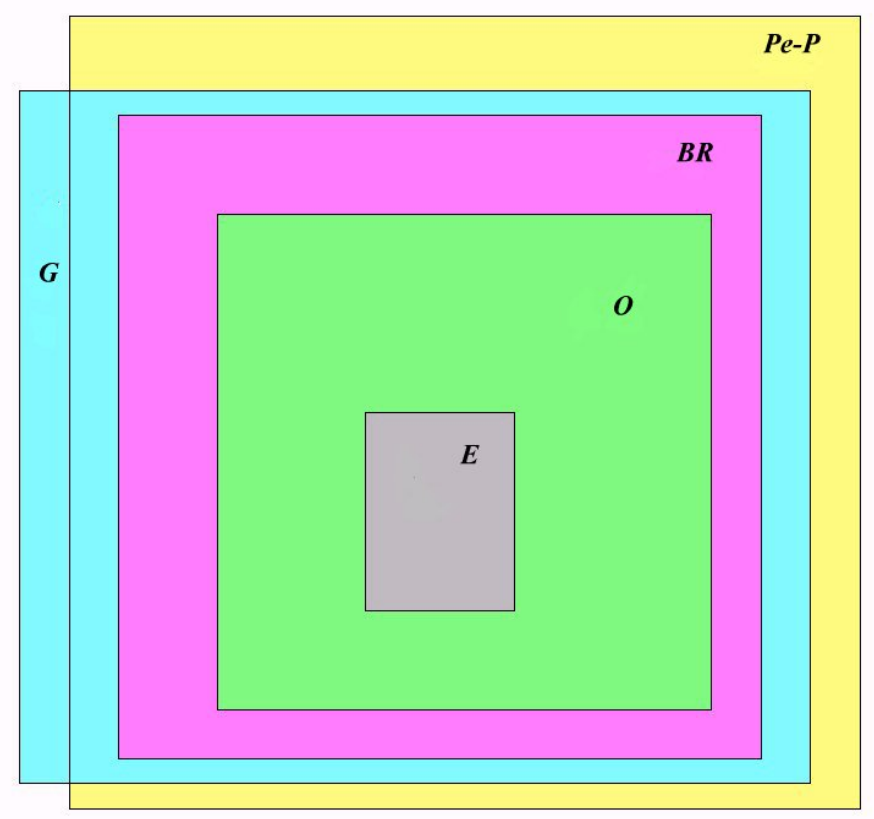




\section{Conclusions and Open Problems}

In this paper some classes of potential games have been studied: generalized, best reply and Pareto potential.

The importance of these games is that they have at least an equilibrium in pure strategy and it corresponds to a Pareto equilibrium of the potential function (generalized, best Pareto, Pareto potential, respectively). Furthermore, these have interesting applications in real life: e.g., network models, environment problems and telecommunication models. For application in the scalar case, see: $[6,11,22]$.

We have studied some properties of these classes but much more may still be investigated, for example:

(1) The study of approximate equilibria for infinite games (see [1,23] for different concepts of approximate equilibria).

(2) The study of the properties of equilibrium with improvement set as introduced in [12], notion which captures contemporary the idea of exact and approximate equilibrium.

(3) The FIP has relation with Pareto equilibria and approximate FIP ( $a$ FIP for short) could be defined for a multicriteria setting and we could study the relations with approximate Pareto equilibria.

(4) It could be interesting to investigate some well posedeness properties of the potential game $G$ via the well posedness of the potential function ([13], for exact potential games in the scalar case and [23] in a multicriteria setting).

(5) Other classes of potential games may be defined and investigated.

(6) Some applications to network and telecommunication problems and environmental models could be investigated via potential games $([6,11])$.

(7) The potential games could be defined via a preorder on the strategy set and interesting properties could be found (see [15]).

Some of these issues are work in progress.

Acknowledgments: The author gratefully thanks the anonymous referees for their helpful comments and suggestions to improve the paper.

Conflicts of Interest: The author declares no conflict of interest.

\section{References}

1. Patrone, F.; Pusillo, L.; Tijs, S. Multicriteria games and Potentials. TOP 2007, 15, 138-145.

2. Pusillo, L. Interactive Decisions and Potential Games. J. Glob. Optim. 2008, 40, 339-352.

3. Rosenthal, R.W. A class of games possessing pure strategy Nash equilibria. Int. J. Game Theory 1973, 2, 65-67.

4. Monderer, D.; Shapley, L.S. Potential games. Games Econ. Behav. 1996, 14, 124-143.

5. Ehrgott, M. Multicriteria Optimization, 2nd ed.; Springer: Berlin/Heidelberg, Germany, 2005.

6. Lã, Q.D.; Chew, Y.H.; Soong, B.-H. Potential Game Theory: Applications in Radio Resource Allocation; Springer: Berlin/Heidelberg, Germany, 2016.

7. Buzzi, S.; Zappone, A. Potential games for energy-efficient resource allocation in multipoint-to-multipoint CDMA wireless data networks. Phys. Commun. 2013, 7, 1-13.

8. Moragrega, A.; Closas, P.; Ibars, C. Potential games for energy-efficient RSS-based positioning in wireless sensor networks. IEEE J. Sel. Area Commun. 2015, 33, 1394-1406.

9. Sagratella, S. Algorithms for generalized potential games with mixed-integer variables. Comput. Optim. Appl. 2017, doi:/10.1007/s10589-017-9927-4.

10. Facchinei, F.; Piccialli, V.; Sciandrone, M. Decomposition algorithms for generalized potential games. Comput. Optim. Appl. 2011, 50, 237-262.

11. Mallozzi, L.; Tijs, S. Conflict and cooperation in symmetric potential games. Int. Game Theory Rev. 2008, 10, 245-256.

12. Pusillo, L.; Tijs, S. E-equilibria for multicriteria games. Adv. Dyn. Games 2012, 12, 217-228.

13. Margiocco, M.; Pusillo, L. Potential games and well posedness properties. Optimization 2008, 57, 571-579.

14. Voorneveld, M. Best response potential games. Econ. Lett. 2000, 66, 289-295. 
15. Voorneveld, M. Potential Games and Interactive Decisions with Multiple Criteria; Dissertation series n.61; Center of Economic Research-Tilburg University: Tilburg, The Netherlands, 1999.

16. Anand, L.; Shashishekhar, N.; Ghose, D.; Prasad, U.R. A survey of solution concept in multicriteria games. J. Indian Inst. Sci. 1995, 75, 141-174.

17. Borm, P.; van Megen, F.; Tijs, S. A perfectness concept for multicriteria games. Math. Methods Oper. Res. 1999, 49, 401-412.

18. Corley, H.W. Games with vector payoff. J. Optim. Theory Appl. 1984, 104, 47-52.

19. Shapley, L.S. Equilibrium points in games with vector payoffs. Nav. Res. Logist. Q. 1959, 6, 57-61.

20. Milchtaich, I. Congestion Games with Player Specific Payoff Functions. Games Econ. Behav. 1996, 3, 82-100.

21. Garcia, F.R.F.; Albandoz, J.P. Teoria de Juegos Multiobjetivo; Universidad de Sevilla: Sevilla, Spain, 2006.

22. Mallozzi, L.; Pusillo, L.; Tijs, S. Approximate equilibria for Bayesian games. JMAA 2007, 342, 1098-1102.

23. Morgan, J. Approximations and Well-Posedness in Multicriteria Games. Ann. Oper. Res. 2005, 137, $257-268$.

(c) 2017 by the author. Licensee MDPI, Basel, Switzerland. This article is an open access article distributed under the terms and conditions of the Creative Commons Attribution (CC BY) license (http:/ / creativecommons.org/licenses/by/4.0/). 